\title{
TEXTURE EVOLUTION IN CHANNEL-DIE COMPRESSION PART II: EFFECTS OF GRAINS WHICH SHEAR
}

\author{
R. BECKER and L. A. LALLI \\ Alcoa Laboratories, Alcoa Center, PA 15069
}

\section{INTRODUCTION}

In a previous study ${ }^{1}$, a finite element model was used to examine the influence of grain interactions on deformation patterns and texture evolution in plane strain compression. In that analysis it was assumed that plastic deformation resulted from slip on crystallographic planes. The model accounted for finite deformations and lattice rotations. Compatibility and equilibrium were satisfied by the finite element method. Local grain interactions produced complex deformation patterns and stress fields. The resulting crystallographic texture was compared to predictions from a Taylor type $\mathrm{e}^{2,3}$ polycrystal texture evolution model.

Another use for such detailed models is in studying the neighboring grain interactions of specific grains or specific groups of grains in order to better understand their behavior in a polycrystal. A particular grain orientation of interest is the Brass texture component which is typically present in rolled products (e.g. the review article by Hirsch and Lücke ${ }^{4}$. This orientation is of interest for rolling processes since many of the simple polycrystal texture evolution models do not predict a strong Brass texture in plane strain compression ${ }^{5,6}$. A grain with a Brass orientation tends to deform with a longitudinal-transverse shear when subjected to plane strain compression. It has been suggested that restrictions on this shear, which are typically imposed in many polycrystal texture evolution models, may impede formation of the Brass texture ${ }^{5,6}$.

In the previous work ${ }^{1}$ it was demonstrated that the detailed polycrystal model does tend to predict a Brass texture. The mechanism of formation is similar to that proposed by Aernoudt and Stüwe ${ }^{7}$ : grains split into regions in which cooperative longitudinal-transverse shearing between the segments of the grain satisfied the boundary conditions on average. The orientations of these grain segments often evolved as complementary texture components. The splitting of grains into distinct regions was controlled by interactions with the neighboring grains.

In this study calculations have been performed using the previously developed model. One of the grains is replaced by a grain of Brass orientation to determine its effects on the model predictions.

\section{NUMERICAL MODEL}

The model region is a portion of an idealized polycrystal depicted in Fig. 1. The grains are hexagonal prisms with the deformation and stress being uniform through the thickness, $H$. Reflective periodic boundary conditions are imposed to maintain compatibility and equilibrium with adjacent material. All of the model surfaces are taken to be planes of reflective symmetry. These faces remain 
planar, and the shear tractions vanish pointwise on the surfaces. The model is deformed in plane strain compression by applying uniform velocity to the surface $X_{3}=H$ such that the through-thickness compressive strain rate is constant. The material extends along the $X_{1}$ direction and $X_{2}$ is the direction of zero strain.

Crystal constitutive relations have been implemented in the finite element code ABAQUS ${ }^{8,9}$. The constitutive model and the numerical integration scheme are identical to the rate dependent crystal constitutive model described by Peirce, Asaro and Needleman ${ }^{10}$. The model accounts for finite strains and rotations as well as for the evolution of texture with deformation. The material properties used in the analyses were obtained from room temperature uniaxial compression experiments on commercial purity aluminum ${ }^{1}$. The slip system hardening was estimated from the stress-strain behavior of the polycrystalline material using a calculated Taylor factor. All slip systems are assumed to harden equally.

Deformation of the region is simulated using the finite element model. The finite element mesh used to discretize the region shown in Fig. 1 is uniform with 24 quadrilaterals along the $X_{2}$ direction and 60 along $X_{1}$, Fig. 2a. Each quadrilateral is composed of four generalized plane strain, constant strain, triangular elements arranged in a "crossed triangle" configuration. Associated with each element are internal variables characterizing the crystal lattice orientation, critical resolved shear stress and the slip system hardening rate for that element. These parameters evolve differently within each element so that property variations can develop within grains.

The initial crystal orientations associated with each grain are based on measured grain orientations within a small region taken from an extruded bar of commercial purity aluminum ${ }^{1}$. These orientations are assigned to the model grains as indicated in Fig. 2b. Neighboring grains with similar shading are of the same initial orientation.

For the present calculations, the orientation of grain 21 has been replaced by a grain with the exact Brass orientation with plane (011) along the compression axis, $X_{3}$, and direction [211] along the direction of extension, $X_{1}$. All other parameters in the model remained the same as in the previous work ${ }^{1}$. Grain 21 was selected as the replacement site since this grain did not shear noticeably and deformed nearly homogeneously in the previous calculation. In plane strain compression, a crystal with this Brass orientation tends to produce a positive longitudinal-transverse shear, so some differences in the solutions are anticipated. To facilitate comparison with results of the previous analysis ${ }^{1}$, deformed finite element meshes at $\varepsilon=0.5$ and $\varepsilon=0.1$ from the previous calculation are given in Figs. 2c and $2 \mathrm{~d}$.

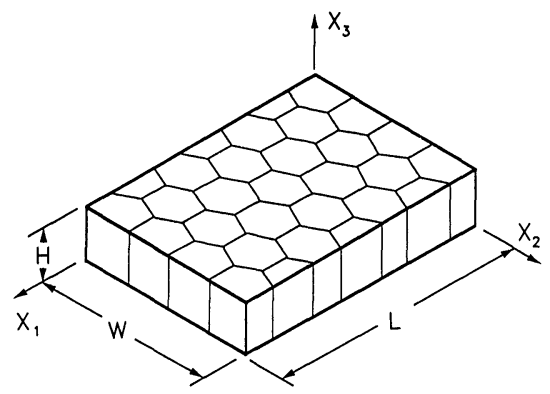

Figure 1. Model geometry showing specimen orientation and idealized grains. 


\section{RESULTS}

Deformed meshes for the calculation with a grain of Brass orientation at grain 21 are given in Fig. 3 at $\varepsilon=0.5$ and $\varepsilon=1.0$. Detailed comparison of Fig. 3 to Fig. 2 shows that about half of the grains exhibited different deformation patterns in the presence of the Brass grain.

a)

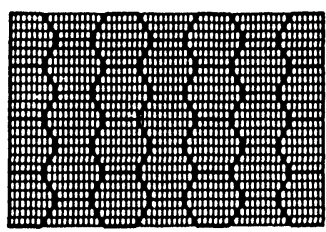

$\varepsilon=0.0$

c)

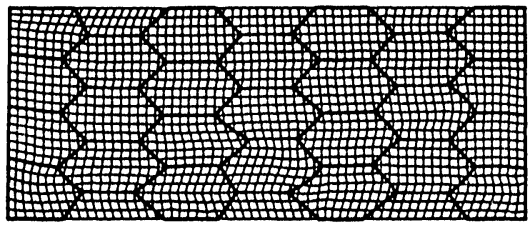

b)

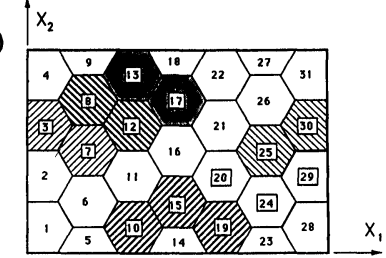

$\varepsilon=0.5$

d)

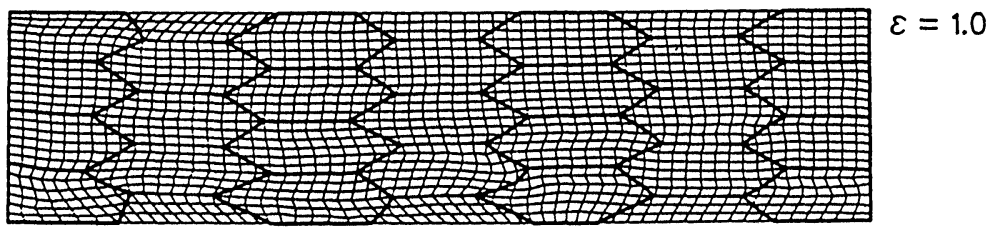

Figure 2. Initial mesh (a), grain distribution (b), and deformed finite element meshes from [1]

a)

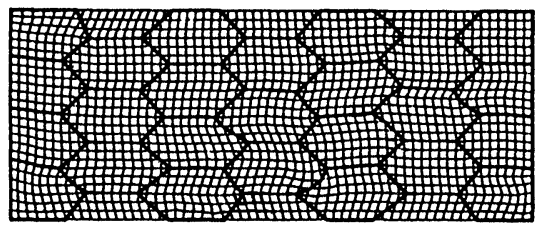

$\varepsilon=0.50$

b)

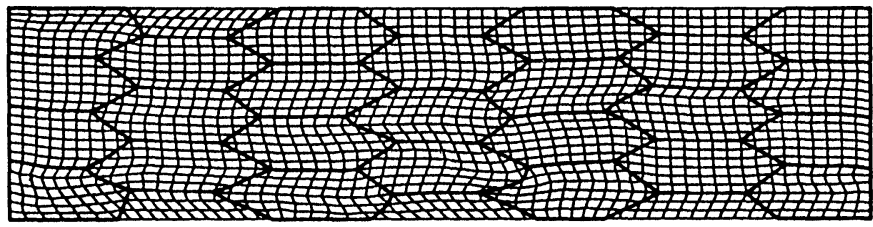

$\varepsilon=1.00$

Figure 3. Deformed finite element meshes for the calculation with an implanted grain of Brass orientation at grain 21 . 
At small strains in the present calculation grain 21 tended to rotate counterclockwise to accommodate the shear strain. However, as the strain increased the rotation ceased and the grain deformed nonuniformly. As the grain lengthened, the angle that the top and bottom grain boundaries make with the $X_{1}$ axis decreased. This is a geometrical effect related to the extension of the grain which tends to decrease the apparent rotation of the grain. As deformation progressed, grain 21 continued to shear, and grains 20 and 22 accommodated the deformation by a combination of shear and rigid displacement in the $X_{1}$ direction. Given the severity of the distortion of grain 21 it might be expected that these neighboring grains would be severely distorted also; however, they are not. Grains 20 and 22 have sheared very little, although grain 20 has rotated. Due to the translation of the grains in the $X_{1}$ direction, the accommodating deformation is spread over several grains across the width. This is facilitated by the shear free boundary conditions which allow the grains to translate along $X_{1}$.

The greatest disturbance caused by. grain 21 is to the neighbors in the $X_{1}$ direction where compatibility requirements transmit the shear. This is evident by comparing the deformation of grain 16 (Fig. 3) to the predictions from the previous calculation (Fig. 2) where this grain deformed uniformly. In the present calculation, grain 16 is so badly distorted that its deformation affects that of grains 11 and 12. The effects of end shear are not as dramatic for all grains. Grains 25 and 26 are also at an end of the Brass grain, but they do not exhibit as severe distortion. Possible reasons for the different behavior may be related to their crystallographic orientation or to their proximity to the straight end boundary at $X_{1}=L$ which imposes regularity on the deformation.

The overall predicted texture evolution for the calculation with the implanted grain of Brass orientation is similar to that of the previous calculation except for a slightly greater shift of intensities from near the Goss orientation $((011)[100])$ toward the $\beta$-fiber which contains the implanted grain of Brass orientation. (The $\beta$-fiber ${ }^{4}$ referred to here is a range of crystal orientations extending from near the Copper component ((11)2)[111]), through the "S" component to the Brass component at $((011)[2 \overline{1} 1])$.) This trend, although slight, suggests that the shear caused by the Brass grain tends to induce the grains to rotate toward the same variant of Brass texture as the implanted grain.

The orientation of grain 21 remains stable despite significant variations in the macroscopic strain throughout the grain. Some orientation spread occurs within the grain as a small rotation about the compression axis, $X_{3}$. This spread is driven by material rotations and in-plane shear stresses. At small strains the bulk of the poles rotated a few degrees away from the ideal orientation in the same direction as the macroscopic rotation of the grain (counter-clockwise). The poles tend to return toward the ideal orientation at larger strains.

Taylor model calculations for plane strain compression including the Brass grain (results not shown) give essentially the same texture prediction as the previous ${ }^{1}$ Taylor model calculation-except, of course, for the different orientation of grain 21. Due to the number of constraints on the Taylor model in plane strain deformation (all but one component of the deformation gradient is prescribed), the grains deform independently except for the requirement that the average stress in the extension direction be zero. Hence, replacing one grain in the model makes little difference.

In the detailed model, the effects of the substitution of the Brass grain for grain 21 are more severe in some grains than in others. The orientation distributions were plotted for individual grains and compared with the previous predictions. While the lattice orientations in most grains were not significantly different, there was a trend of a greater shift toward a Brass texture that was reflected in the overall texture. The crystal lattice in several grains, however, was dramatically affected by the presence of the implanted grain. A few of these cases are described below. 

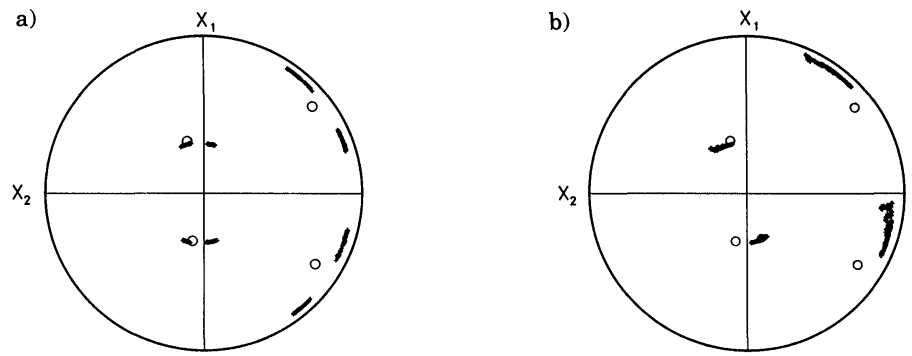

Figure 4. (111) pole figure of grain 20 at $\varepsilon=1.0$ (a) from the previous calculation ${ }^{1}$ and (b) from the model with the implanted Brass grain.

A comparison of Figs 2 and 3 indicates that grain 20 sheared differently as a result of the introduction of grain 21 . The predicted lattice rotation is also significantly different from the previous predictions. In the previous calculation, the grain split into two distinct grain fragments, Fig. 4a. Here, the grain does not split and the direction of lattice rotation is in the direction of the material rotation, Fig. 4b. The spread of orientations from the grain boundary at grain 19 to the grain boundary at grain 21 provides a transition from near Goss toward Brass. The orientation developing near grain 21 is evolving toward the same branch of the $\beta$-fiber which contains grain 21 . However, the shear deformation is opposite to that of grain 21. This creates a situation where the grains shear in opposite directions, but the lattice orientations are similar. It has been suggested that this phenomenon is related to the strength of the shear stress from the Brass grain compared to the resistance offered by its neighbors ${ }^{11}$. These calculations were not carried out far enough to determine the result of this transient behavior.

In the previous calculation ${ }^{1}$ grain 16 deformed nearly homogeneously, and the lattice rotation and the orientation spread were very small. Here, however, the shear imposed by grain 21 caused grain 16 to split into two dominant grain fragments, Fig. 5. While both the upper and lower grain fragments of grain 16 sheared approximately the same amount (Fig. 3), only the lower one showed considerable lattice rotation. The orientation of the upper fragment has considerable spread, but remains near the cube orientation, similar to the previous results. An average orientation of the lower grain fragment is near the " $\mathrm{S}$ " location. This rotation moved the lattice in the lower grain fragment toward the $\beta$-fiber containing grain 21 .
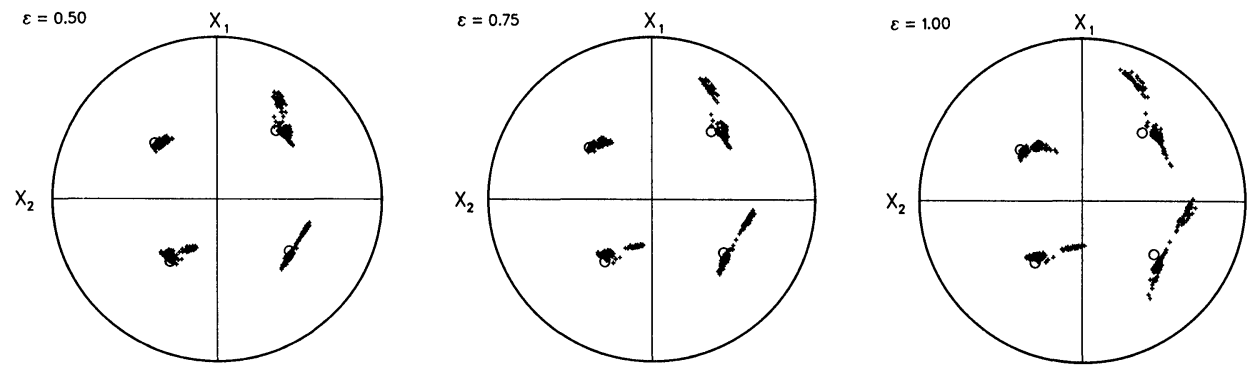

Figure 5. Texture evolution for grain 16, open circles mark the initial orientation. 

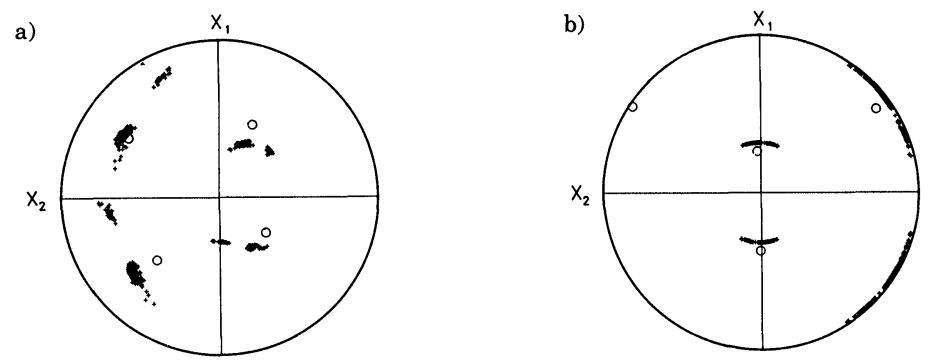

Figure 6. (111) pole figures of (a) grain 25 and (b) grain 26 at $\varepsilon=1.0$.

Grains 25 and 26 are at locations similar to grain 16 with respect to grain 21 . In grain 25 at $\varepsilon=1.0$, Fig. $6 \mathrm{a}$, the orientation of the lattice in the top row of elements rotated in the direction of the material rotation (clockwise). This deformation was driven by grain 21 . The remainder of the grain remained near its initial orientation near the $\langle 100\rangle$ fiber along the $X_{1}$ axis. Grain 26 split into two grain fragments with the orientation spread at $\varepsilon=1.0$, Fig. $6 \mathrm{~b}$, being much wider than in the models without the implanted Brass grain. The lattice in the lower two rows of elements rotated toward the $\beta$-fiber not containing the implanted Brass grain, and the bulk of the grain has orientations spread around Goss ((011)[100]).

\section{CONCLUSIONS}

Comparison of the present results with those of a previous analysis ${ }^{1}$ illustrates the effects of a particular grain orientation on the deformation patterns and texture evolution of the surrounding grains. The crystallographic orientation of grains with strong textures, such as Brass, can be stable even if the strain is nonhomogeneous. Other orientations, such as Cube, are easily perturbed.

The present results indicate that the presence of a strongly shearing grain, e.g. a Brass grain, can induce neighboring grains located in a direction normal to the shearing direction to shear in the opposite sense. Although the final stable configuration is not determined here, these calculations suggest that this induced shear will not necessarily result in complementary orientations.

\section{REFERENCES}

1 R. Becker, Acta Metall., in press.

2 G. I. Taylor, J. Inst Metals, 62, 307 (1938).

3 R. J. Asaro and A. Needleman, Acta Metall., 33, 923 (1985).

4 J. R. Hirsch and K. Lücke, Acta Metall., 36, 2863 (1988).

5 H. Mecking, Proc. ICOTOM 6, Tokyo, The iron and steel inst. of Japan (1981).

6 J. R. Hirsch and K. Lücke, Acta Metall., 36, 2883 (1988).

7 E. Aernoudt and H. P. Stüwe, Z. Metallk., 61, 128 (1970).

8 ABAQUS Users' Manual, version 4.7, Hibbitt Karlsson and Sorensen, Providence (1988).

9 R. E. Smelser and R. Becker, Proceedings of the ABAQUS Users' Conference, 207, Stresa, Italy (1989).

10 D. Peirce, R. J. Asaro and A. Needleman, Acta Metall. 30, 1087 (1982).

11 R. Becker, J. Mech. Phys. Solids, in press. 